\title{
Synchronization and Inverse Synchronization of Some Different Dimensional Discrete-time Chaotic Dynamical Systems via Scaling Matrices
}

\author{
Adel Ouannas \\ LAMIS Laboratory, Department of Mathematics and Computer Science, \\ University of Tebessa, 12002, Algeria.
}

\begin{abstract}
In this paper, new types of synchronization and inverse synchro-nization are proposed for some diaerent dimensional chaotic dynamical systems in discrete-time using scaling matrices. Based on Lyapunov stability theory and nonlinear controllers, new synchronization results are derived. Numerical simulations are used to verify the eaectiveness of the proposed schemes.
\end{abstract}

\section{Keyword}

Synchronization, inverse synchronization, chaotic dynamical sys-tems, discrete-time, Lyapunov stability

\section{Introduction}

Dynamical systems in discrete-time play an important role in chaos theory and mathematical modelisation of many scienti.c problems [1, 2, 3, 4]. Re-cently, more and more attention has been paid to the synchronization of chaos(hyperchaos) in discrete-time dynamical systems, due it.s applications in se- cure communication and cryptology [5, 6]. Many synchronization types have been found $[7,8,9]$ and diaerent methods are used to study synchronization of discrete-time chaotic systems $[10,11,12]$.

In this paper, the proplems of synchronization with scaling matrix and it.s inverse type are studied between drive-response chaotic systems in discrete-time. Based on Lyapunov stability theory, we would like to present a con-structive schemes to investigate synchronization and inverse synchronization between some typical chaotic dynamical systems with respect to scaling matrices in discrete-time with diaerent dimensions. Because in real world all chaotic maps are described by plane equations or space systems, we restrict our study about the new chaos synchronization types to $2 \mathrm{D}$ and $3 \mathrm{D}$ discrete chaotic sys-tems and this restriction does .n lose the generality of our main results. Firstly, anew schemes are proposed to study synchronization and inverse synchronization between the drive 2D Lorenz discrete-time system and the response 3D Wang map. Secondly, the 3D generalized Hénon map is considered as the drive system and the controlled Fold map as the response system to achieve synchronization and inverse 
synchronizationThe remainder of this paper is organized as follows. In Section 2, de.n-itions of synchronization and inverse synchronization for discrete systems via scaling matrices are introduced. In section 3; synchronization and inverse syn-chronization are applied to 2D drive system and 3D response system and new synchronization results are derived. In Section 4, synchronization and inverse synchronization are studied between 3D drive system and 2D response system.Finally, the paper is concluded in Section 5.

\section{Definitions of synchronization and inverse syn-chronization via scaling matrices}

Consider the following drive chaotic system described by

Consider the following drive chaotic aystem described by

$$
F(k+1)=f(x(k))
$$

where $x(k)=\left(x_{1}(k), \ldots, x_{n}(k)\right)^{T} \in \mathbb{R}^{n}$ is the atate vector of the drive system and $f: \mathbb{R}^{n} \rightarrow \mathbb{R}^{n}$. As the respone system, we consider the following chaotic syatem described by

$$
Y(k+1)=g(Y(k))+U
$$

where $Y(t)=\left(y_{1}(k), \ldots, y_{m}(k)\right)^{T} \in \mathbb{R}^{m}$ is the atate vector of the response system, $g: \mathbb{R}^{m} \rightarrow \mathbb{R}^{m}$ and $U=\left(u_{i}\right)_{1 \leq \leq m} \in \mathbb{R}^{m}$ if the vector controller to be determined.

We present the definition of aynchroniation via acaling matrix for coupled chaotic syatems given in Eqs. (1) and (2).

Definition 1 The drive system (1) and the rsponse system (2) are said to be syuchronized, with respect to the scaling motrix $A$, if there exsits os controller $U=\left(u_{4}\right)_{1 \leq s m} \in \mathbb{R}^{m}$ and a given matrix $A, m \times n$, sueh that the synehronisation error

$$
e(B)=Y(B)-A Y(B)
$$

satisfes that $\lim _{k \rightarrow+\infty}\|e(k)\|=0$.

The definition of inwerse synchroniagtion vis scaling matrix for coupled chatic aystema giwen in Eqs. (1) and (2) is given next.

Definition 2 The drive system (1) and the respone system (2) are said to be inerse synehronized, with respect to the seating motmin $\theta$, if there exists a controller $U=\left(u_{1}\right)_{1 \leq \mathrm{s} m} \in \mathbb{R}^{m}$ and o given motris $\theta, n \times m$, such thot the s ymehronizotion error

$$
e(k)=x(k)-\theta Y(k)
$$

sotisfues thot $\lim _{k \rightarrow+\infty}\|e(k)\|=0$. 
International Journal of Chaos, Control, Modelling and Simulation (IJCCMS) Vol.3, No.4, December 2014

\section{Synchronization and inverse synchronization of 2D drive system and $3 D$ response system}

In this section, we consider Lorenz discrete-time system and as the drive system the controlled Wang system. Lorenz discrete-time system can be described as

$$
\left\{\begin{array}{c}
x_{1}(k+1)=(1+a b) x_{1}(k)-b x_{1}(k) x_{2}(k) \\
x_{2}(k+1)=(1-b) x_{2}(k)+b x_{1}^{2}(k)
\end{array}\right.
$$

which has a chaotic attractor, for ex ample, when $(a, b)=(1.25,0.75)$ [12]. The Lorenz discrete-time chsotic attractor is shown in Fig. 1.

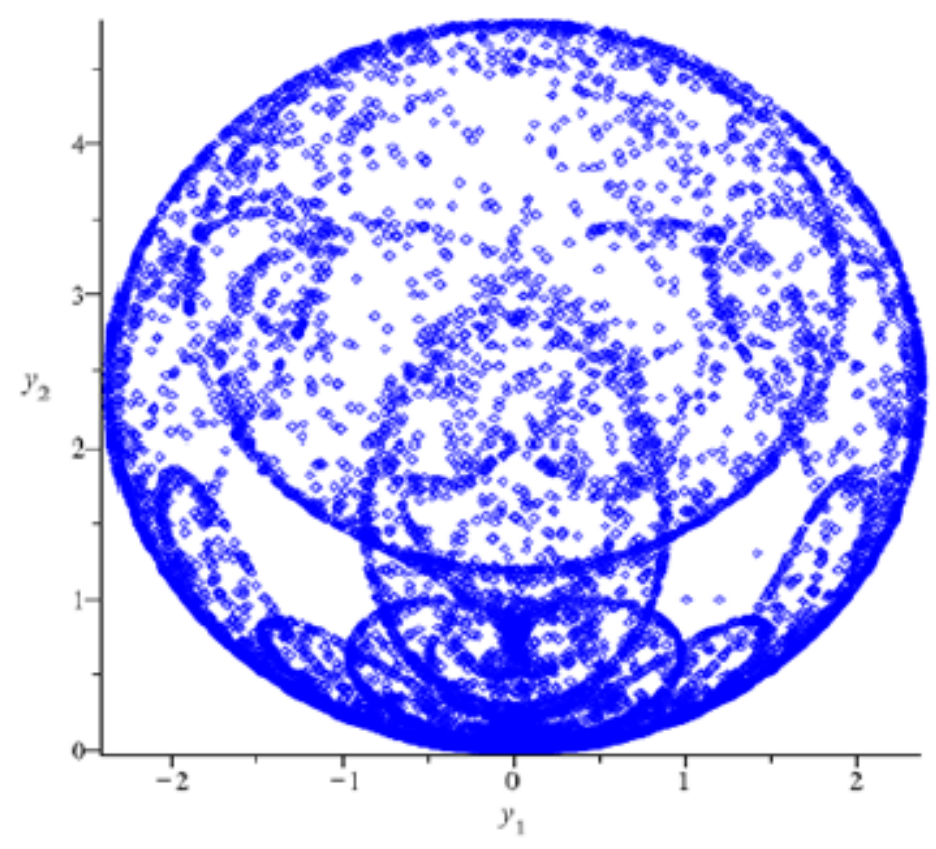

Fig. 1 The chaotic attractor of Lorenz discrete-time system.

The controlled Wang aystem can be described as

$$
\left\{\begin{array}{c}
y_{1}(k+1)=a_{3} b_{2}(k)+\left(a_{4} \delta+1\right) y_{1}(k)+u_{1}, \\
y_{2}(k+1)=a_{1} b_{1}(k)+y_{2}(k)+a_{2} \delta y_{3}(k)+u_{2}, \\
y_{3}(k+1)=\left(a_{7} \delta+1\right) y_{3}(k)+a_{6} b_{2}(k) y_{3}(k)+a_{5} \delta+u_{3}
\end{array}\right.
$$

where $U=\left(u_{1}, u_{2}, u_{3}\right)^{T}$ is the vector controller. The Wang discrete-time gyatem has a chatic attractor, for example, when $\left(a_{1}, a_{2}, a_{3}, a_{4}, a_{5}, a_{6}, a_{7}, 0\right)=$ $(-1.9,0.2,0.5,-2.3,2,-0.6,-1.9,1)[12]$. the chaotic attractor of Wang discrete- 


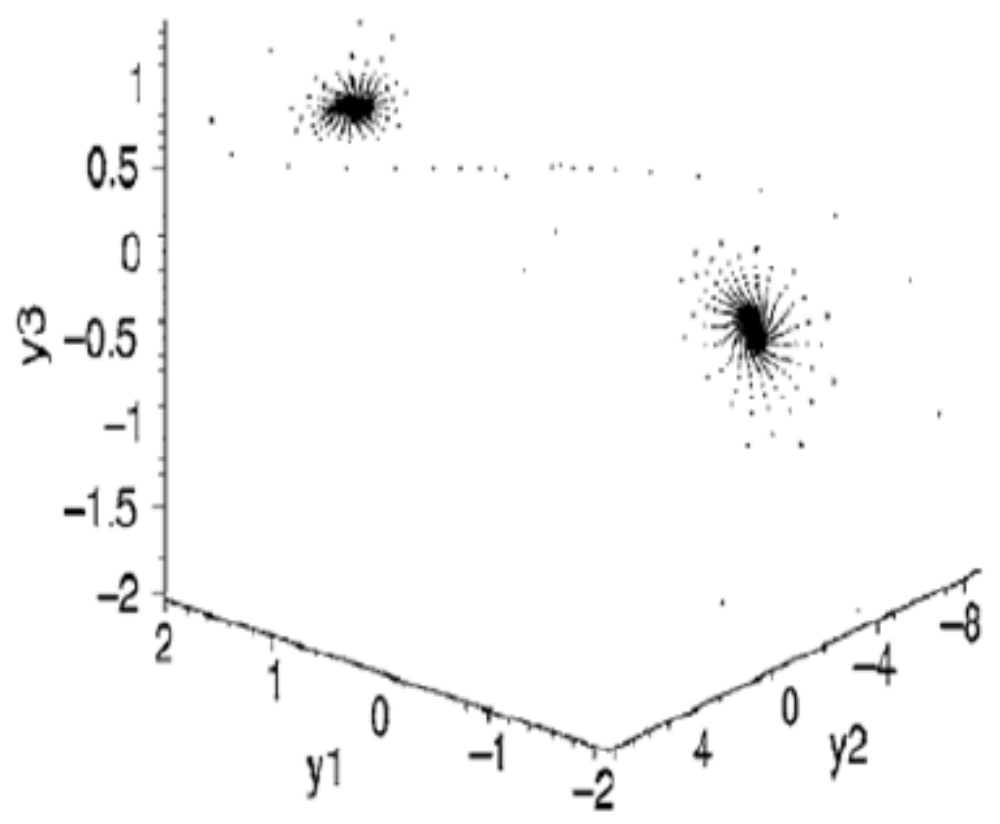

Fig. 2 The chaotic attractor of Wang discrete-time aystem

\subsection{Synchronization of Lorenz discrete-time system and Wang system}

According to de ntion 1, the synchronization errors between the drive system (5) and the response system (6) can be derived as

$$
\left\{\begin{array}{c}
e_{1}(k+1)=\left(a_{1} \delta+1-l_{1}\right) e_{1}(k)+L_{2}+N_{1}+u_{1} \\
e_{2}(k+1)=e_{2}(k)+L_{2}+N_{2}+u_{2} \\
e_{3}(k+1)=\left(a_{7} \delta+1-l_{2}\right) e_{3}(k)+L_{3}+N_{3}+u_{3}
\end{array}\right.
$$

where $l_{1}, l_{2}$ are real control constanta to be detrmined,

$$
\begin{aligned}
& \left\{\begin{array}{c}
N_{1}=h_{11} b x_{1}(k) x_{2}(k)-h_{12} b x_{1}^{2}(k) \\
N_{2}=h_{21} b x_{1}(b) x_{2}(k)-h_{22} b x_{1}^{2}(k) \\
N_{3}=a_{6} b y_{2}(k) y_{3}(k)+a_{7} b+h_{31} b x_{1}(k) x_{2}(k)-A_{32} b x_{1}^{2}(k)
\end{array}\right. \\
& \left\{\begin{array}{c}
L_{1}=b_{1 y_{1}}(k)+a_{3} \delta_{y_{2}}(k)+\sum_{j=1}^{2} w_{1 j} x_{j}(k) \\
L_{2}=a_{1} \delta_{1}(k)+a_{3} \delta_{3}(k)+\sum_{j=1}^{2} w_{2 j} x_{j}(k) \\
L_{3}=\sum_{j=1}^{2} \omega_{3 j} x_{j}(k)
\end{array}\right.
\end{aligned}
$$




$$
\left\{\begin{array}{c}
\omega_{11}=h_{11}\left(a_{4} \delta-a b-l_{1}\right) \\
\omega_{12}=h_{12}\left(a_{11} \delta+b-l_{1}\right) \\
\omega_{21}=-h_{21} a b \\
\omega_{22}=h_{22} b \\
\omega_{31}=A_{31}\left(a_{7} \delta-a b-l_{2}\right) \\
\omega_{32}=h_{32}\left(a_{7} \delta+b-l_{2}\right)
\end{array}\right.
$$

and $A=\left(A_{j}\right) \in \mathbb{R}^{3 \times 2}$ is the scaling matrix.

Theorem 3 If $l_{1}$ and $l_{2}$ are ehosen such that

$$
\left|\alpha_{4} \delta+1-l_{1}\right|<1 \text { ard }\left|\alpha_{7} \delta+1-l_{2}\right|<1 .
$$

Then, the drive system ( 5 ) and the response system ( 6 ) are globalty synchronized, with respect to the orbitrory seding motis $h$, under the followith controllers

$$
u_{i}=-L_{i}-N_{i}, \quad 1 \leq i \leq 3 .
$$

Froof. By substituting the control lew (12) into (7), the gynchroniagtion error can be written as

$$
\left\{\begin{array}{c}
e_{1}(k+1)=\left(a_{4} \delta+1-b_{1}\right) e_{1}(k) \\
e_{2}(k+1)=e_{2}(k) \\
e_{3}(k+1)=\left(a_{7} \delta+1-b_{3}\right) e_{3}(k)
\end{array}\right.
$$

We take as a candidate Lyapunow funotion:

$$
V(e(k))=\sum_{j=1}^{3} e_{j}^{2}(k)
$$

we get:

$$
\begin{aligned}
\Delta V(e(k)) & =V(e(k+1))-V(e(k)) \\
& =\sum_{j=1}^{3} e_{j}^{2}(k+1)-\sum_{j=1}^{3} e_{i}^{2}(k) \\
& =\left(\left(a_{1} \delta+1-l_{1}\right)^{2}-1\right) e_{1}^{2}(k)+\left(\left(a_{7} \delta+1-l_{2}\right)^{2}-1\right) e_{3}^{2}(k)
\end{aligned}
$$

and by using (11), we obtain: $\triangle V(e(k))<0$. Thus, by Lyapunov atability it is immediate that $\lim _{k \rightarrow \infty} e_{i}(k)=0,(i=1,2)$, and from the fact $\lim _{k \rightarrow \infty}\|e(k)\|=$ 0 . We conclude that the aystema (5) and (6) are globally generalized anchronized. 
The error functions evolution are shown in Fig. 3.

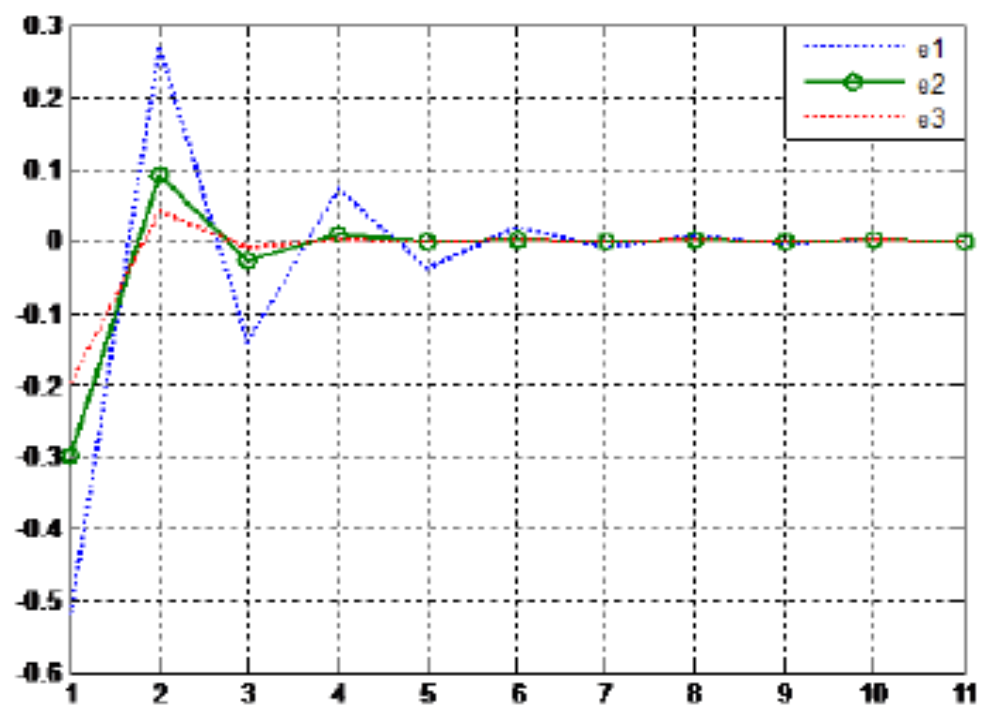

Fig. 3 Time evolution of aynchronization errors between the drive Lorenz discrete-time system and the response Wang system.

\subsection{Inverse synchronization between Lorenz discrete-time system and Wang system}

According to de ntion 2, the synchronization errors between the drive system (5) and the response system (6)), can be derived as 
International Journal of Chaos, Control, Modelling and Simulation (IJCCMS) Vol.3, No.4, December 2014

$$
\left\{\begin{array}{c}
e_{1}(k+1)=\left(1+a b-i_{1}\right) e_{1}(k)+R_{1}-\sum_{j=1}^{3} b_{1 j} u_{j} \\
e_{2}(k+1)=\left(1-b-l_{2}\right) e_{2}(b)+R_{2}-\sum_{j=1}^{\theta} \theta_{2 j} u_{j}
\end{array}\right.
$$

where $l_{1}, l_{2}$ are real contanta to be detrmined,

$$
R_{i}=L_{i}+N_{i}, \quad i=1,2,
$$

where

$$
\begin{gathered}
\left\{\begin{array}{c}
N_{1}=-b x_{1}(b) x_{2}(b)-\theta_{13} a_{5} \delta y_{2}(k) y_{3}(k)-\theta_{13} a_{5} \delta \\
N_{2}=b x_{1}^{2}(b)-\theta_{23} a_{6} \delta y_{2}(k) y_{3}(b)-\theta_{23} a_{5} \delta
\end{array}\right. \\
\left\{\begin{array}{l}
L_{1}=\sum_{j=1}^{3} \omega_{1 j} y_{j}(k) \\
L_{2}=\sum_{j=1}^{3} \omega_{2 j} y_{j}(k)
\end{array}\right.
\end{gathered}
$$

where

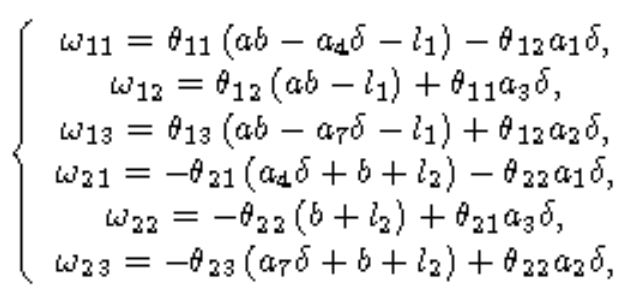

and $\theta=\left(\theta_{i j}\right) \in \mathbb{R}^{2 \times 3}$ is the scaling matrix

To achieve synchonization between the driwe syatem (b) and the response gyatem (6), we asaume that

$$
\theta_{11} \theta_{22} \neq \theta_{12} \theta_{21}
$$

and we choose the controllers $u_{i},(1 \leq i \leq 3)$, as follow

$$
u_{1}=\frac{\theta_{12} R_{2}-\theta_{22} R_{1}}{\theta_{11} \theta_{22}-\theta_{12} \theta_{21}}, u_{2}=\frac{\theta_{21} R_{1}-\theta_{11} R_{2}}{\theta_{11} \theta_{22}-\theta_{12} \theta_{21}} \text { and } u_{3}=0 \text {. }
$$


International Journal of Chaos, Control, Modelling and Simulation (IJCCMS) Vol.3, No.4, December 2014

Theorem 4 If $l_{1}$ and $l_{3}$ are ehosen sueh that

$$
\left|1+a b-l_{1}\right|<1 \text { and }\left|1-b-l_{2}\right|<1 .
$$

Then, the drive system (b) and the response system ( $\theta$ ) are globaly inverse s ynehronized, with respeet to the scaling matrix of which verifes (20), under the eontrollers.(21).

Froof. By substituting the controllers (21) into (15), the synch ronization errors can be written as

$$
\left\{\begin{array}{c}
e_{1}(k+1)=\left(1+a b-l_{1}\right) e_{1}(k) \\
e_{2}(k+1)=\left(1-b-l_{2}\right) e_{2}(k)
\end{array}\right.
$$

We take as a candidate Lyapunor function:

$$
V(e(k))=\sum_{j=1}^{2} e_{j}^{2}(k)
$$

we get:

$$
\begin{aligned}
\Delta V(e(k)) & =V(e(k+1))-V(e(k)) \\
& =\sum_{j=1}^{2} e_{i}^{2}(k+1)-\sum_{j=1}^{2} e_{i}^{2}(k) \\
& =\left(\left(1+a b-l_{1}\right)^{2}-1\right) e_{1}^{2}(k)+\left(\left(1-b-l_{2}\right)^{2}-1\right) e_{1}^{2}(k)
\end{aligned}
$$

and by using (22), we obtain: $\Delta V(e(k))<0$. Thus, by Lyapunov atability it is immediate that $\lim _{k \rightarrow \infty} e_{i}(k)=0,(i=1,2)$, and from the fact $\lim _{k \rightarrow \infty}\|e(k)\|=$ 
0 . We conclude that the systems (5) and (6) are globally inverse synchronized.

The error functions evolution are shown in Fig. 4.

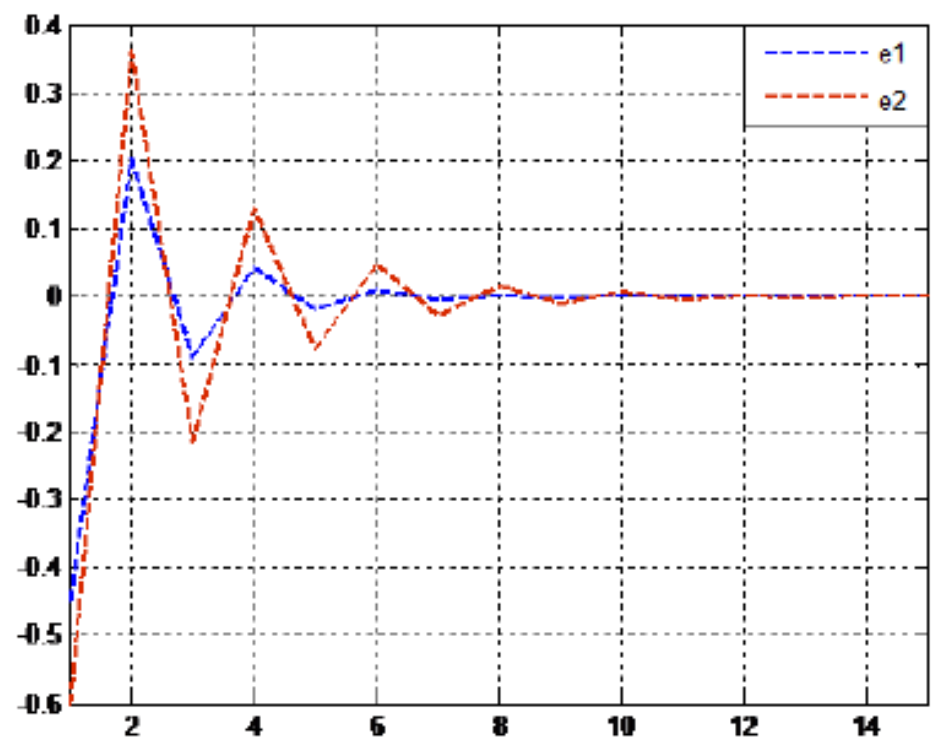

Fig. 4 Time evolution of inverse synchronization errors between the drive Lorenz discrete-time system and the response Wang system.

\section{Synchronization and inverse synchronization of 3D drive system and 2D response system}

Now, we consider 3D generalized Hénon map as the drive system and the con- trolled Fold map as the response system. The 3D generalized Hénon map can be described as

$$
\left\{\begin{array}{c}
x_{1}(k+1)=-\beta x_{2}(k) \\
x_{2}(k+1)=x_{3}(k)+1-\alpha x_{2}^{2}(k) \\
x_{3}(k+1)=x_{1}(k)+\beta x_{2}(k)
\end{array}\right.
$$


which has a chaotic attractor, for example, when $(\alpha, \beta)=(1.07,0.3)$ [11]. The $3 \mathrm{D}$ generalized Henon chaotic attractor is shown in Fig. 4.

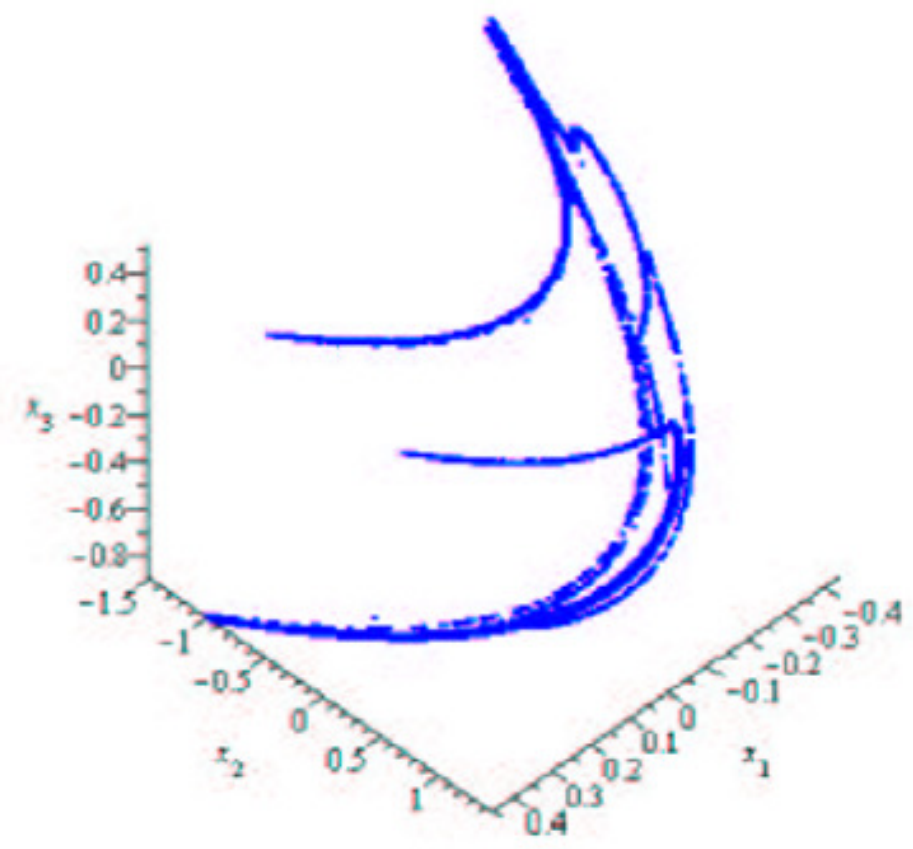

Fig. 5 The chaotic attractor of $3 \mathrm{D}$ generalized Henon.

The controlled Fold map can be described as

$$
\left\{\begin{array}{c}
y_{1}(k+1)=a y_{1}(k)+y_{2}(k)+u_{1}, \\
y_{2}(k+1)=y_{1}^{2}(k)+b+u_{2},
\end{array}\right.
$$

where $U=\left(u_{1}, u_{2}\right)^{T}$ is the vector controller. The Fold map has a chatic attractor, for example, when $(a, b)=(-0.1,-1.7)[12]$. the chaotic attractor of 
Fold map is shown in Fig. 6.

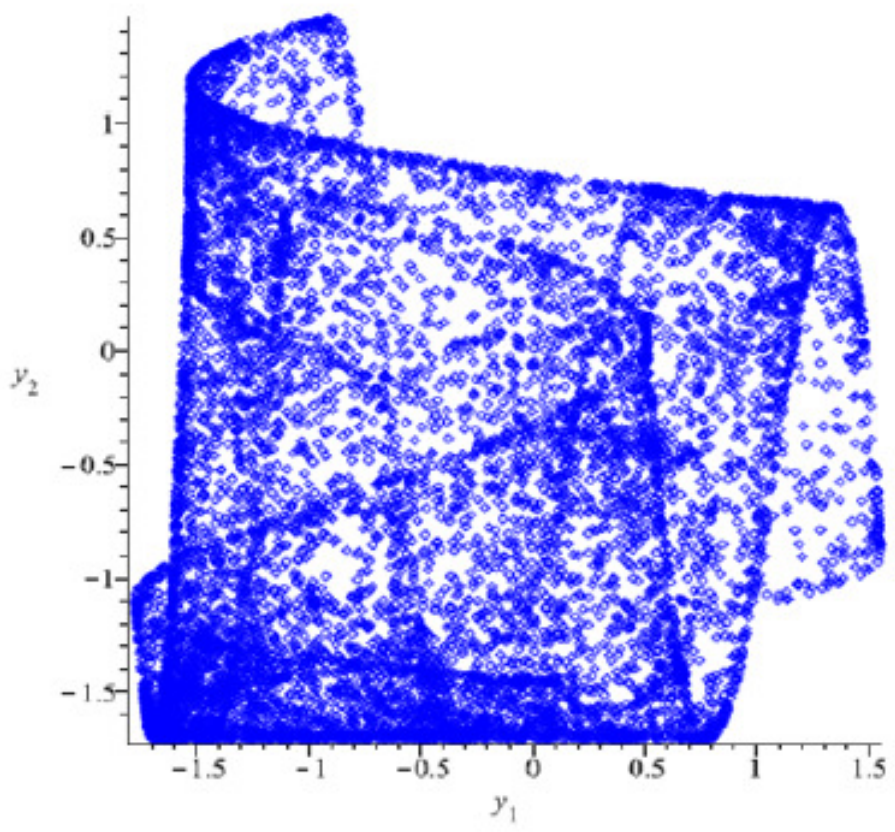

Fig. 6 The chaotic attractor of Fold map.

\subsection{Synchronization between 3D generalized Hénon map and Fold map}

According to de ntion 1, the synchronization errors between systems (25) and (26), can be derived as

$$
\left\{\begin{array}{c}
e_{1}(k+1)=\left(a-l_{1}\right) e_{1}(k)+M_{i}+L_{i}+u_{1} \\
e_{2}(k+1)=e_{2}(k)+N_{i}+L_{i}+u_{2}
\end{array}\right.
$$

where $l$ is a real constant to be detrmined,

$$
\begin{gathered}
\left\{\begin{array}{c}
N_{1}=-h_{12}\left(1-a x_{2}^{2}(k)\right) \\
N_{2}=y_{1}^{2}(k)+b-h_{22}\left(1-a x_{2}^{2}(k)\right)
\end{array}\right. \\
\left\{\begin{array}{c}
L_{1}=y_{2}(k)+\sum_{j=1}^{3} \gamma_{1 j} x_{j}(k) \\
L_{2}=-y_{2}(k)+\sum_{j=1}^{3} \gamma_{2 j} x_{j}(k)
\end{array}\right.
\end{gathered}
$$

where 


$$
\left\{\begin{array}{c}
\gamma_{11}=h_{11}\left(a-l_{1}\right)-h_{13}, \\
\gamma_{12}=h_{12}\left(\alpha+\beta-l_{1}\right)-h_{13} \beta \\
\gamma_{13}=h_{13}\left(a-l_{1}\right)-h_{12} \\
\gamma_{21}=h_{21}-h_{23} \\
\gamma_{22}=h_{22}+h_{21} \beta-h_{23} \beta \\
\gamma_{23}=h_{23}-h_{22}
\end{array}\right.
$$

and $h=\left(h_{j}\right) \in \mathbb{R}^{2 \times 3}$ is the acaling matrix.

Theorem 5 If the control constant $l$ is ehosen sueh that

$$
|b-l|<1
$$

Then, the drive system (25) and the respone system (26) are globaly synehrorized, respect to the orbitrory sociting motrix $\mathrm{A}$, under the following controlders

$$
u_{i}=-N_{i}-L_{i}, \quad 1 \leq i \leq 2 .
$$

Froof. By aubstituting Eq. (32) into (27), the aynchronization errors can be written as

$$
\left\{\begin{array}{c}
e_{1}(k+1)=(a-l) e_{1}(k) \\
e_{2}(k+1)=e_{2}(b)
\end{array}\right.
$$

We take as a candidate Lyapunor function

$$
V(e(k))=\sum_{j=1}^{2} e_{j}^{2}(k)
$$

we get:

$$
\begin{aligned}
\Delta V(e(k)) & =V(e(k+1))-V(e(k)) \\
& =\sum_{j=1}^{2} e_{i}^{2}(k+1)-\sum_{j=1}^{2} e_{i}^{2}(k) \\
& =\left((b-l)^{2}-1\right) e_{1}^{2}(k)
\end{aligned}
$$

and by using (31), we obtain: $\Delta V(e(k))<0$. Thus, by Lyapunow atability it is immedia te that $\lim _{k \rightarrow \infty} e_{i}(k)=0,(i=1,2)$, and from the fact $\lim _{k \rightarrow \infty}\|e(k)\|=$ 0 . We conclude that the syatems (25) and (26) are globaly aynchronized. 
We get the numeric result that is shown in Fig. 7 .

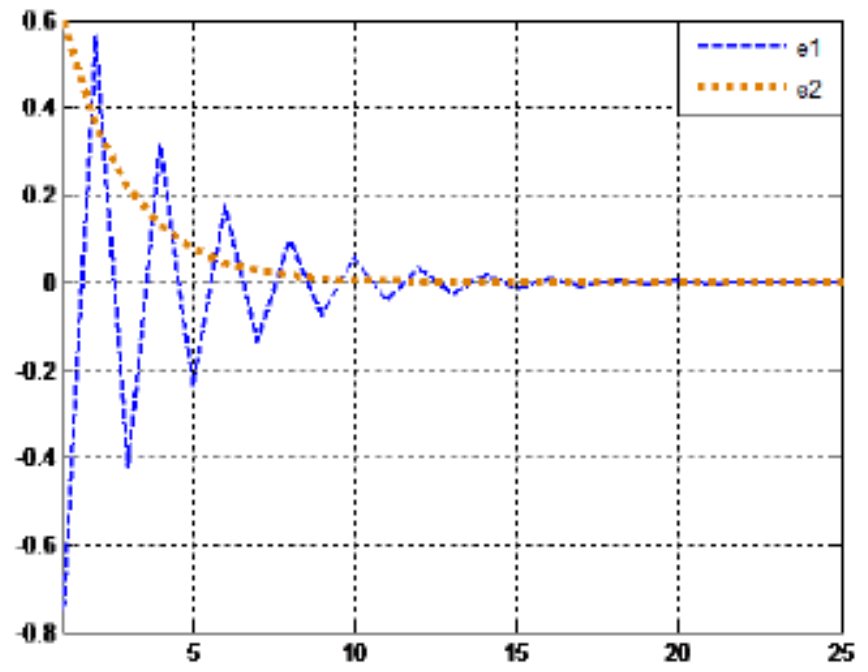

Fig. 7 Time evolution of aynchronization errors between the drive $3 \mathrm{D}$ generalized Henon map and the response Fold map.

\subsection{Inverse synchronization between 3D generalized Hénon map and Fold map}

In this case, the synchronization errors between the drive system (25) and the response system (26) can be derived as 
International Journal of Chaos, Control, Modelling and Simulation (IJCCMS) Vol.3, No.4, December 2014

$$
\left\{\begin{array}{c}
e_{1}(k+1)=(-\beta+b) e_{2}(k)+R_{1}-\sum_{j=1}^{2} \theta_{1 j} u_{j} \\
e_{2}(k+1)=e_{3}(k)+R_{2}-\sum_{j=1}^{2} \theta_{2 j} u_{j} \\
e_{3}(k+1)=e_{1}(k)+R_{3}-\sum_{j=1}^{\theta_{3 j} u_{j}}
\end{array}\right.
$$

where $l$ ia a real control constant to be determined,

$$
R_{i}=r_{i}+L_{i}, \quad i=1,2,3
$$

where

$$
\left\{\begin{array}{c}
N_{1}=-\theta_{12}\left(y_{1}^{2}(k)+b\right) \\
N_{2}=1-\alpha x_{2}^{2}(k)-\theta_{22}\left(y_{1}^{2}(k)+b\right) \\
N_{3}=-\theta_{32}\left(y_{1}^{2}(k)+b\right) \\
L_{1}=\sum_{j=1}^{3} \mu_{3 j} x_{j}(k) \\
L_{2}=\sum_{j=1}^{3} \mu_{3 j} x_{j}(k) \\
L_{3}=\beta_{2}(k)+\sum_{j=1}^{3} \mu_{3 j} x_{j}(k)
\end{array}\right.
$$

where

$$
\left\{\begin{array}{c}
\mu_{11}=\theta_{11}(l-\beta-\alpha), \\
\mu_{12}=(-\beta+l) \theta_{12}-\theta_{11} \\
\mu_{21}=\theta_{31}-\theta_{21} a \\
\mu_{22}=\theta_{32}-\theta_{21} \\
\mu_{31}=\theta_{11}-\theta_{31} a_{3} \\
\mu_{32}=\theta_{12}-\theta_{31}
\end{array}\right.
$$

and $\theta=\left(\theta_{i j}\right) \in \mathbb{R}^{2 \times 3}$ is the acaling matrix.

To achieve aynchonization between the drive syatem (25) and the reaponae sytem (26), we asume that

$$
\theta_{12} \theta_{21} \theta_{31}-2 \theta_{11} \theta_{31} \theta_{22}+\theta_{11} \theta_{21} \theta_{32} \neq 0
$$

and we chooge the controllerg $u_{i},(1 \leq i \leq 2)$, as follow

$$
u_{1}=\frac{-\theta_{12} \theta_{31} R_{1}+2 \theta_{22} \theta_{31} R_{2}-\theta_{21} \theta_{32} R_{3}}{\theta_{12} \theta_{21} \theta_{31}-2 \theta_{11} \theta_{31} \theta_{22}+\theta_{11} \theta_{21} \theta_{32}},
$$

and

$$
u_{2}=\frac{-\theta_{21} \theta_{31} R_{1}+2 \theta_{11} \theta_{31} R_{2}-\theta_{11} \theta_{21} R_{3}}{\theta_{12} \theta_{21} \theta_{31}-2 \theta_{11} \theta_{31} \theta_{22}+\theta_{11} \theta_{21} \theta_{32}} .
$$


International Journal of Chaos, Control, Modelling and Simulation (IJCCMS) Vol.3, No.4, December 2014

Theorem 6 If the control constant $l$ is ehosen such that

$$
|\beta-l|<1
$$

Then, the drive system (25) and the response system (26) are globaly inverse synchro nized, with respect to the seating matris is wich verifes (40), under the control Lows (41) and (42)

Froof. By substituting Eqs. (41) and (42) into (35), the synehronization error can be written as

$$
\left\{\begin{array}{c}
e_{1}(k+1)=(-\beta+l) e_{2}(k) \\
e_{2}(k+1)=e_{3}(k) \\
e_{3}(k+1)=e_{1}(k)
\end{array}\right.
$$

We take as a candidate Lyapunor function:

$$
V(e(k))=\sum_{j=1}^{3} e_{j}^{2}(k)
$$

we get:

$$
\begin{aligned}
\Delta V(e(b)) & =V(e(k+1))-V(e(b)) \\
& =\sum_{j=1}^{3} e_{i}^{2}(k+1)-\sum_{j=1}^{3} e_{i}^{2}(b) \\
& =\left((\beta-l)^{2}-1\right) e_{2}^{2}(b)
\end{aligned}
$$


and by using (41), we obtain: $\Delta V(e(k))<0$. Thus, by Lyapunov atability it is immediate that $\lim _{k \rightarrow \infty} e_{i}(k)=0,(i=1,2)$, and from the fact $\lim _{k \rightarrow \infty}\|e(k)\|=$ 0 . We conclude that the aystems (25) and (26) are globally inverse generalized synchronized.

Finally, we get the numeric result that is shown in Fig. 8.

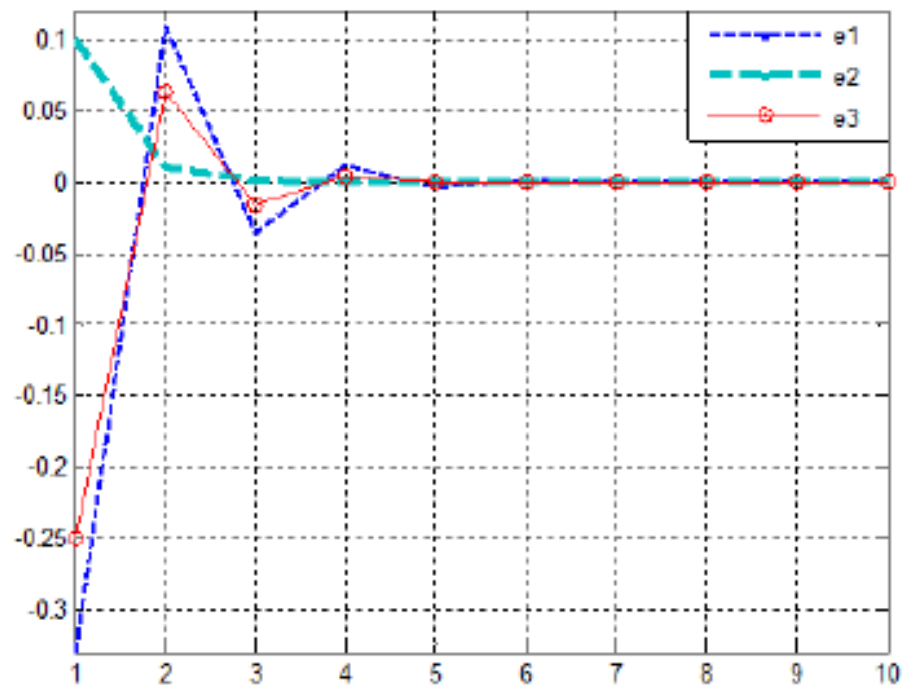

Fig. 8 Time evolution of inverse synchronization errors between the drive $3 \mathrm{D}$ generalized Henon map and the response Fold map.

\section{Conclusion}

In this paper, we analysed the synchronization and the inverse synchronization problems using scaling matrices for some typical diaerent dimensional chaotic systems in discrete-time. A new control schemes are derived and new synchro-nization controllers are proposed. Numerical simulations are used to verify the exectiveness of the derived results. 
International Journal of Chaos, Control, Modelling and Simulation (IJCCMS) Vol.3, No.4, December 2014

\section{References}

[1] A. M. Selvam. Nonlinear Dynamics and Chaos: Applications in At-mospheric Sciences. J. Adv. Math. Appl. 1, 181-205 (2012)

[2] J. Lei. Stochastic Modeling in Systems Biology. J. Adv. Math. Appl. 1,76-88 (2012)

[3] Y. Wang and G. Fariello. On Neuroinformatics: Mathematical Models of Neuroscience and Neurocomputing. J. Adv. Math. Appl. 1, 206-217 (2012)

[4] X. Quan, Y. Lu, F. Xu, J. Lei, and W. Liu. Mathematical Modeling of Question Popularity in UserInteractive Question Answering Systems J.Adv. Math. Appl. 2, 24-31 (2013)

[5] E. Solak. Cryptanalysis of observer based discrete-time chaotic encryptionschemes. Inter. J. Bifur. Chaos. 15(2), 653-658 (2005)

[6] Liu, W., Wang, Z.M. and Zhang, W.D. Controlled synchronization of discrete-time chaotic systems under communication constraints. Nonlinear Dyn. 69, $223230(2012)$

[7] A. Ouannas. Co-existence of Complete Synchronization and Anti-Synchronization in a Class of Discrete Rational Chaotic Systems. Far East.

J. Dyn. Syst. 23(1-2), 41-48 (2014)

[8] A. Ouannas. A New Q-S Synchronization Scheme for Discrete Chaotic Sys-tems. Far East. J. Appl. Math. 84(2), 89-94 (2013)

[9] A. Ouannas. On Full-State Hybrid Projective Synchronization of General Discrete Chaotic Systems. J. Nonl. Dyn Volume 2014

[10] G. Grassi. Generalized synchronization between diøerent chaotic maps via dead-beat control. Chin. Phys. B. 21(5), 050505 (2013).

[11] Y. Zhenya. Q-S synchronization in 3D Hénon-like map and generalized Hénon map via a scalar controller. Phys. Lett. A. 342, 309-317 (2005).

[12] Y. Zhenya. Q-S (complete or anticipated) synchronization backstepping scheme in a class of discretetime chaotic (hyperchaotic) systems: A symbolic-numeric computation approach. Chaos 16, 01311911 (2006). 